\title{
Novel therapeutic strategies for multiple sclerosis: potential of intravenous immunoglobulin
}

\section{Jagadeesh Bayry, Sébastien Lacroix-Desmazes \& Srini V. Kaveri}

In a recent article (Novel therapeutic strategies for multiple sclerosis - a multifaceted adversary. Nature Rev. Drug Discov. 7, 909925; 2008) ${ }^{1}$, Lopez-Diego and Weiner discuss a number of novel potential therapies to treat multiple sclerosis (MS) that target leukocyte trafficking, T cells and B cells. However, the authors do not discuss an important immunotherapeutic option for relapsing-remitting MS (RRMS): intravenous immunoglobulin (IVIg) - a therapeutic preparation of normal human polyclonal immunoglobulin $\mathrm{G}$ obtained from pools of plasma from several thousand healthy blood donors.

IVIg has been extensively used to treat a wide range of autoimmune and inflammatory disorders of neuromuscular and systemic origin ${ }^{2,3}$. Several randomized, double-blind studies in RRMS have shown a beneficial effect of IVIg on disease with respect to reductions in relapse rates and lesion activity detected with magnetic resonance imaging $^{4-9}$. Although a recent study did not find a beneficial effect of IVIg in RRMS ${ }^{10}$, for several reasons (discussed below), we believe that IVIg remains a reasonable therapeutic candidate for RRMS and could yet prove its therapeutic utility, provided some unresolved issues on its clinical use can be addressed.

The therapeutic dose of IVIg is empirically set at $2 \mathrm{~g}$ per $\mathrm{kg}$, which is infused either in five daily doses of $400 \mathrm{mg}$ per $\mathrm{kg}$ each, or divided into two or three daily doses ${ }^{2,3}$. The studies with IVIg in RRMS have involved different dosages, and patients with different stages of disease and previous treatment histor $\mathrm{y}^{4-7}$, indicating that the treatment regimen for IVIg for RRMS has yet to be optimized. Indeed, a recent European Federation of Neurological Societies task force recommended IVIg as a second- or third-line therapy for RRMS, but did not include it among first-line therapies owing to the limited evidence for clinical efficacy and optimum dosage ${ }^{11}$. Therefore, the first challenge would be to establish the minimum effective dose and the precise dosing interval of IVIg for induction and maintenance of immune tolerance in RRMS.

Therapy of MS with monoclonal antibodies (mAbs) is associated with several adverse effects and the immune tolerance is not long lasting. In addition, the pathogenesis of MS cannot be attributed to a single cell type or cytokine. In this context, the multiple and overlapping mechanisms of action of IVIg and its proven safety could provide a new option for a combination therapy with $\mathrm{mAbs}^{2,3}$. Indeed, the combination of the antiCD20 mAb rituximab (Rituxan/MabThera; Biogen Idec/Genentech/Roche) and IVIg has been found to be effective in refractory pemphigus vulgaris and transplantation ${ }^{12,13}$. Such a combination therapy might not only reduce the dose of both mAbs and IVIg, thereby reducing the potential for adverse effects, but could also impart a more long-lasting therapeutic effect compared with monotherapy ${ }^{14}$.

In conclusion, we consider that the available data indicate that IVIg is not a lost cause for the therapy of $\mathrm{MS}^{15,16}$, and that clinical trials are therefore warranted to determine if a combination of mAbs with IVIg could lead to a more effective therapy for RRMS.

Jagadeesh Bayry, Sébastien Lacroix-Desmazes and Srini V. Kaveri are at the Institut Nationale de la Santé et de la Recherche Médicale, U872, Paris, F-75006, France; The Centre de Recherche des Cordeliers, Equipe 16-Immunopathology and therapeutic immunointervention, Université Pierre et Marie Curie Paris 6, UMR S 872, 15 Rue de l'Ecole de Médicine, Paris, F-75006, France; and the Universite Paris Descartes, UMR S 872, Paris, F-75006, France. e-mail: jagadeesh.bayry@crc.jussieu.fr
Lopez-Diego, R. S. \& Weiner, H. L. Novel therapeutic strategies for multiple sclerosis - a multifaceted adversary. Nature Rev. Drug Discov. 7, 909-925 (2008).

2. Bayry, J., Lacroix-Desmazes, S., Kazatchkine, M. D. ¿ Kaveri, S. V. Monoclonal antibody and intravenous immunoglobulin therapy for rheumatic diseases: rationale and mechanisms of action. Nature Clin. Pract. Rheumatol. 3, 262-272 (2007).

3. Gold, R., Stangel, M. \& Dalakas, M. C. Drug insight: the use of intravenous immunoglobulin in neurology - therapeutic considerations and practical issues. Nature Clin. Pract. Neurol. 3, 36-44 (2007).

4. Fazekas, F., Deisenhammer, F., Strasser-Fuchs, S., Nahler, G. \& Mamoli, B. Randomised placebo-controlled trial of monthly intravenous immunoglobulin therapy in relapsing-remitting multiple sclerosis. Austrian Immunoglobulin in Multiple Sclerosis Study Group. Lancet 349 589-593 (1997)

5. Achiron, A. et al. Intravenous immunoglobulin treatment in multiple sclerosis. Effect on relapses. Neurology 50, 398-402 (1998).

6. Sorensen, P. S. et al. Intravenous immunoglobulin C reduces MRI activity in relapsing multiple sclerosis. Neurology 50, 1273-1281 (1998).

7. Lewanska, M., Siger-Zajdel, M. \& Selmaj, K No difference in efficacy of two different doses of intravenous immunoglobulins in MS: clinical and MRI assessment. Eur. J. Neurol. 9, 565-572 (2002).

8. Achiron, A. et al. Intravenous immunoglobulin treatment following the first demyelinating event suggestive of multiple sclerosis: a randomized, double-blind, placebo-controlled trial. Arch. Neurol. 61, 1515-1520 (2004)

9. Haas, J., Maas-Enriquez, M. \& Hartung, H. P. Intravenous immunoglobulins in the treatment of relapsing remitting multiple sclerosis - results of a retrospective multicenter observational study over five years. Mult. Scler. 11, 562-567 (2005).

10. Fazekas, F. et al. Intravenous immunoglobulin in relapsing-remitting multiple sclerosis: a dose-finding trial. Neurology 71, 265-271 (2008)

11. Elovaara, l. et al. EFNS guidelines for the use of intravenous immunoglobulin in treatment of neurological diseases: EFNS task force on the use of intravenous immunoglobulin in treatment of neurological diseases. Eur. J. Neurol. 15, 893-908 (2008).

12. Ahmed, A. R., Spigelman, Z., Cavacini, L. A. \& Posner, M. R. Treatment of pemphigus vulgaris with rituximab and intravenous immune globulin. N. Engl. J. Med. 355, 1772-1779 (2006).

13. Vo, A. A. et al. Rituximab and intravenous immune globulin for desensitization during renal transplantation. N. Engl. J. Med. 359, 242-251 (2008).

14. Bayry, J., Kazatchkine, M. D. \& Kaveri, S. V. Shortage of human intravenous immunoglobulinreasons and possible solutions. Nature Clin. Pract. Neurol. 3, 120-121 (2007).

15. Fazekas, F., Strasser-Fuchs, S. \& Hommes, O. R. Intravenous immunoglobulin in MS: promise or failure? J. Neurol. Sci. 259, 61-66 (2007)

16. Cohen, J. A. How effective is intravenous immunoglobulin for the treatment of relapsing remitting multiple sclerosis? Nature Clin. Pract Neurol. 4, 588-589 (2008).

\section{Acknowledgements}

Supported by grants from Institut National de la Santé et de la Recherche Médicale and Centre National de la Recherche Scientifique (INSERM), Centre National de la Recherche Scientifique (CNRS), Université Paris Descartes - Paris VI, and Université Pierre et Marie Curie - Paris VI. 